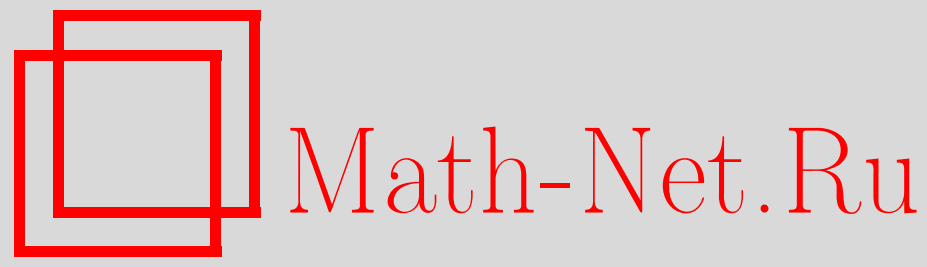

А. Р. Миротин, Об отображении совместного спектра набора генераторов полугрупп, Функи. анализ и его прил., 2012, том 46, выпуск 3, 62-70

DOI: https://doi.org/10.4213/faa3076

Использование Общероссийского математического портала MathNet.Ru подразумевает, что вы прочитали и согласны с пользовательским соглашением

http://www . mathnet.ru/rus/agreement

Параметры загрузки:

IP : 54.224 .135 .184

26 апреля 2023 г., 16:23:40

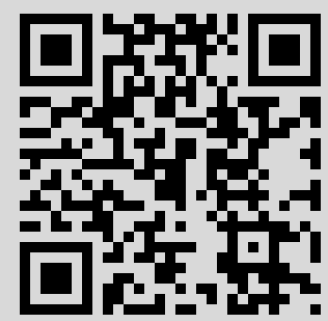


Функциональный анализ и его приложения

2012, т. 46, вып. 3, с. $62-70$

УДК 517.983.23

\title{
Об отображении совместного спектра набора генераторов полугрупп*
}

\author{
(c) 2012. А. Р. Миротин
}

В рамках многомерного функционального исчисления генераторов полугрупп, основанного на классе функций Бернштейна нескольких переменных («многомерного функционального исчисления Бохнера-Филлипса»), устанавливается теорема об отображении спектра Тейлора набора коммутирующих генераторов.

\section{§1. Введение и предварительные сведения}

Одномерное функциональное исчисление Бохнера-Филлипса является существенной частью теории полугрупп операторов (см., например, [1]-[5]) и находит важные применения в теории случайных процессов (см. [6, гл. XIII, §7], [7, гл. XXIII, §5], [8]). Основы многомерного исчисления были заложены автором в [9]-[11]. В данной статье мы определяем совместный спектр Тейлора набора коммутирующих генераторов полугрупп операторов класса $C_{0}$ в банаховом пространстве и устанавливаем теорему об отображении этого спектра для многомерного исчисления Бохнера-Филлипса. Эта теорема выводится из теоремы об отображении коммутантного спектра. Для формулировки результатов напомним необходимые понятия и факты из [9] и [11].

Определение 1 [9]. Будем говорить, что неположительная функция $\psi \in$ $C^{\infty}\left((-\infty ; 0)^{n}\right)$ принадлежит классу $\mathscr{T}_{n}$ (или является функцией Бернштейна $n$ переменных), если все ее частные производные первого порядка абсолютно монотонны (функция из $C^{\infty}\left((-\infty ; 0)^{n}\right)$ называется абсолютно монотонной, если она неотрицательна вместе со своими частными производными всех порядков).

Очевидно, что $\mathscr{T}_{n}$ есть конус относительно поточечного сложения функций и умножения на скаляр.

Известно [11], что каждая функция $\psi \in \mathscr{T}_{n}$ допускает интегральное представление (здесь и ниже точкой мы обозначаем скалярное произведение в $\mathbb{R}_{+}^{n}$; запись $s \rightarrow-0$ означает, что $\left.s_{1} \rightarrow-0, \ldots, s_{n} \rightarrow-0\right)$ :

$$
\psi(s)=c_{0}+c_{1} \cdot s+\int_{\mathbb{R}_{+}^{n} \backslash\{0\}}\left(e^{s \cdot u}-1\right) d \mu(u) \quad\left(s \in(-\infty ; 0)^{n}\right),
$$

где $c_{0}=\psi(-0):=\lim _{s \rightarrow-0} \psi(s)$, а $c_{1}$ из $\mathbb{R}_{+}^{n}$ и положительная мера $\mu$ на $\mathbb{R}_{+}^{n} \backslash\{0\}$ определяются по $\psi$ однозначно.

Всюду далее через $T_{1}, \ldots, T_{n}$ будут обозначаться попарно коммутирующие однопараметрические $C_{0}$-полугруппы (т. е. сильно непрерывные на $\mathbb{R}_{+}$полугруппы) в комплексном банаховом пространстве $X$, удовлетворяющие условию $\left\|T_{j}(t)\right\| \leqslant M(t \geqslant 0, M=$ const $)$. Через $A_{j}$ обозначим генератор полугруппы

*Работа выполнена при финансовой поддержке Государственной программы фундаментальных исследований Республики Беларусь, договор № 20061473. 
$T_{j}$ с областью определения $D\left(A_{j}\right)$ и положим $A=\left(A_{1}, \ldots, A_{n}\right)$. Далее коммутирование операторов $A_{1}, \ldots, A_{n}$ означает коммутирование соответствующих полугрупп. Через $\operatorname{Gen}(X)$ мы будем обозначать множество всех генераторов равномерно ограниченных $C_{0}$-полугрупп в $X$, а через $I$ - единичный оператор B $X$.

Операторнозначная функция $T(u):=T_{1}\left(u_{1}\right) \cdots T_{n}\left(u_{n}\right)\left(u \in \mathbb{R}_{+}^{n}\right)$ является $n$-параметрической полугруппой класса $C_{0}$, а потому линейное многообразие $D(A):=\bigcap_{j=1}^{n} D\left(A_{j}\right)$ плотно в $X[7, \S 10.10]$.

Определение 2 [11]. Определим значение функции $\psi$ из $\mathscr{T}_{n}$ вида (1) на наборе $A=\left(A_{1}, \ldots, A_{n}\right)$ при $x \in D(A)$ формулой

$$
\psi(A) x=c_{0} x+c_{1} \cdot A x+\int_{\mathbb{R}_{+}^{n} \backslash\{0\}}(T(u)-I) x d \mu(u),
$$

где $c_{1} \cdot A x:=\sum_{j=1}^{n} c_{1}^{j} A_{j} x$.

Пусть $\psi \in \mathscr{T}_{n}, t \geqslant 0$. Тогда функция $g_{t}(s):=e^{t \psi(s)}$ будет абсолютно монотонной на $(-\infty ; 0)^{n}$. Очевидно также, что $g_{t}(s) \leqslant 1$. В силу многомерного варианта теоремы Бернштейна-Уиддера (см., например, [12]) существует такая единственная ограниченная положительная (вероятностная, если $\psi(0)=0)$ мера $\nu_{t}$ на $\mathbb{R}_{+}^{n}$, что при $s \in(-\infty ; 0)^{n}$

$$
g_{t}(s)=\int_{\mathbb{R}_{+}^{n}} e^{s \cdot u} d \nu_{t}(u) .
$$

Определение 3. Используя обозначения, введенные выше, положим

$$
g_{t}(A) x=\int_{\mathbb{R}_{+}^{n}} T(u) x d \nu_{t}(u) \quad(x \in X)
$$

(интеграл понимается в смысле Бохнера).

Очевидно, что $\left\|g_{t}(A)\right\| \leqslant M^{n}$. Поскольку $g_{t+r}(s)=g_{t}(s) g_{r}(s)$, то $\nu_{t}$ образуют сверточную полугруппу ограниченных мер на $\mathbb{R}_{+}^{n}$. Поэтому $g(A): t \mapsto g_{t}(A)$ есть равномерно ограниченная полугруппа операторов на $X$. В частности, $g(A)$ есть $C_{0}$-полугруппа. В одномерном случае она называется полугруппой, подчиненной nолугруппе $T$ (терминология восходит к теории вероятностей, см. [6, §Х.7], [8]).

В [13] было замечено, что основная теорема из [11] может быть уточнена следующим образом.

Теорема 1. Замыкание оператора $\psi(A)$ существует и является генератором полугруппы $g(A)$ класса $C_{0}$, определенной формулой (4).

В самом деле, в [11] было доказано, что оператор $\psi(A)$ замыкаем и его расширением является генератор $G C_{0}$-полугруппы $g(A)$. Поэтому $\overline{\psi(A)} \subseteq G$ (черта обозначает замыкание оператора). Покажем, что здесь имеет место равенство. Поскольку операторы $g_{t}(A)$ коммутируют с $T_{k}(s)$ при всех $k$, то, как легко проверить, $g_{t}(A): D\left(A_{k}\right) \rightarrow D\left(A_{k}\right)$ при всех $k$, а потому и $g_{t}(A): D(A) \rightarrow D(A)$. Отсюда следует, что $D(A)$ есть существенная область для генератора $G$ (см. [14, следствие 3.1.7]). С другой стороны, $D(A)$ есть существенная область для оператора $\overline{\psi(A)}$, причем сужения операторов $\overline{\psi(A)}$ и $G$ на $D(A)$ совпадают. Поэтому $\overline{\psi(A)}=G$, что и завершает доказательство. 
Предыдущая теорема мотивирует окончательный вариант определения оператора $\psi(A)$.

Определение 4 [11]. Под значением функиии $\psi$ из $\mathscr{T}_{n}$ на наборе $A=$ $\left(A_{1}, \ldots, A_{n}\right)$ коммутирующих операторов из $\operatorname{Gen}(X)$ будем понимать генератор полугруппы $g(A)$. Это значение мы далее обозначаем через $\psi(A)$. Возникающее функциональное исчисление будем называть многомерным исчислением Бохнера-Филлипса или $\mathscr{T}_{n}$-исчислением.

Введенные выше обозначения и ограничения далее будут применяться без дополнительных пояснений.

\section{§2. Определение спектра Тейлора и формулировка основной теоремы}

Уже для функции $\psi \in \mathscr{T}_{1}$ теорема об отображении спектра может не выполняться в том смысле, что равенство $\psi(\sigma(A))=\sigma(\psi(A))$, где $A \in \operatorname{Gen}(X), \sigma(A)$ - спектр оператора $A$, может не иметь места, в чем нас убеждает следующий пример.

Пример 1. Пусть $\psi(s)=e^{s}-1$. В этом случае представляющая мера $\mu$ равна $\varepsilon_{1}$, мере Дирака на $\mathbb{R}$, сосредоточенной в единице. В силу определения 4 и теоремы 1 тогда $\psi(A)=T(1)-I$. Но известно, что существует такая равномерно ограниченная полугруппа $T_{2}$ класса $C_{0}$, для которой $\sigma\left(A_{2}\right)=\varnothing$ (см., например, $\left[15\right.$, пример 9.6]). Тогда $\sigma\left(T_{2}(1)\right) \neq e^{\sigma\left(A_{2}\right)}$, а потому $\sigma\left(\psi\left(A_{2}\right)\right) \neq \psi\left(\sigma\left(A_{2}\right)\right)$.

Определение 5. Обобщенный комплекс Кошуля $\mathscr{K}(X, A)$ набора $A$ определим как последовательность пространств и операторов

$$
0 \longleftarrow X_{0} \stackrel{d_{0}}{\longleftarrow} X_{1} \longleftarrow \cdots \stackrel{d_{n-1}}{\longleftarrow} X_{n} \longleftarrow 0,
$$

где $X_{m}=X \otimes \bigwedge^{m} \mathbb{C}^{n}(m=0, \ldots, n)$, а каждый оператор $d_{m}=d_{m}(A)$ имеет область определения $D\left(d_{m}\right) \subset X_{m+1}$, являющуюся линейной оболочкой векторов вида $x \otimes e_{i_{1}} \wedge \cdots \wedge e_{i_{m+1}}$, где $\left(e_{k}\right)_{k=1}^{n}$ - стандартный базис в $\mathbb{C}^{n}, 1 \leqslant i_{1}<\cdots<$ $i_{m+1} \leqslant n$, а $x \in D\left(A_{j_{1}} \cdots A_{j_{m+1}}\right)$ для любой перестановки $\left(j_{1}, \ldots, j_{m+1}\right)$ мультииндекса $\left(i_{1}, \ldots, i_{m+1}\right)$. Для каждого $m=0, \ldots, n-1$ оператор $d_{m}$ однозначно определяется на $D\left(d_{m}\right)$ равенством

$$
d_{m}\left(x \otimes e_{i_{1}} \wedge \cdots \wedge e_{i_{m+1}}\right)=\sum_{k=1}^{m+1}(-1)^{k} A_{i_{k}} x \otimes e_{i_{1}} \wedge \cdots \wedge \widehat{e_{i_{k}}} \wedge \cdots \wedge e_{i_{m+1}}
$$

(«крышка», как обычно, означает, что стоящий под ней вектор опущен).

Хотя обобщенный комплекс Кошуля формально выглядит так же, как и комплекс Кошуля для набора ограниченных операторов (см., например, [16]), отличие состоит в том, что дифференциалы $d_{m}$ не ограничены. При этом их области определения $D\left(d_{m}\right)$ выбраны, как легко проверить, таким образом, что

$$
d_{m}: D\left(d_{m}\right) \rightarrow D\left(d_{m-1}\right) .
$$

Последовательность $\mathscr{K}(X, A)$ является комплексом в том смысле, что $d_{m-1} d_{m} x=0$ для всех $x \in D\left(d_{m}\right)$ и всех $m$. Это проверяется непосредственно с помощью следующего утверждения.

Лемма 1. Если вектор $x$ принадлежит $D\left(A_{i} A_{j}\right) \cap D\left(A_{i}\right)$, то он принадлежит $D\left(A_{j} A_{i}\right)$ u $A_{i} A_{j} x=A_{j} A_{i} x(i, j=1, \ldots, n)$. 
Доказательство. Лемма прямо следует из теоремы 10.9.4 в [7].

(Последовательность

$$
0 \longleftarrow X_{0} \stackrel{d_{0}}{\longleftarrow} D\left(d_{0}\right) \longleftarrow \cdots \stackrel{d_{n-1}}{\longleftarrow} D\left(d_{n-1}\right) \longleftarrow 0
$$

является комплексом векторных пространств и их морфизмов, но не является комплексом нормированных пространств и их морфизмов в классическом понимании термина «комплекс».)

Все сказанное выше о $\mathscr{K}(X, A)$ остается верным для любого набора $\left(A_{1}, \ldots\right.$, $\left.A_{n}\right)$ неограниченных операторов в $X$, обладающих свойством, сформулированным в лемме 1 . В частности, это относится к набору $\lambda-A:=\left(\lambda_{1} I-\right.$ $\left.A_{1}, \ldots, \lambda_{n} I-A_{n}\right), \lambda \in \mathbb{C}^{n}$, где $A$ (как и всюду в данной работе) - набор попарно коммутирующих генераторов полугрупп, описанный выше. При этом $D\left(d_{m}(\lambda-A)\right)=D\left(d_{m}(A)\right)$ для любого $m$.

Определение 6. Спектр Тейлора $\sigma_{T}(A)$ набора $A$ состоит из тех $\lambda \in \mathbb{C}^{n}$, для которых комплекс $\mathscr{K}(X, \lambda-A)$ не является точным.

Ясно, что при $n=1$ спектр $\sigma_{T}\left(A_{1}\right)$ совпадает с обычным спектром $\sigma\left(A_{1}\right)$ оператора $A_{1}$.

Отметим, что, вообще говоря,

$$
\operatorname{pr}_{1} \sigma_{T}(A) \neq \sigma\left(A_{1}\right)
$$

т. е. проекционное свойство для нашего спектра Тейлора может нарушаться (в качестве примера можно взять $T(u)=T_{2}\left(u_{2}\right)$, где $T_{2}-$ полугруппа из примера 1 ; здесь $\left.\sigma_{T}(A)=\varnothing, \sigma\left(A_{1}\right)=\{0\}\right)$.

Основным результатом работы является следующая

Теорема 2. Для любой функиии Бернштейна $\psi$ от $n$ переменных справедливо включение

$$
\sigma(\psi(A)) \supseteq \psi\left(\sigma_{T}(A)\right) .
$$

При $\psi(s)=e^{t \cdot s}-1\left(t \in \mathbb{R}_{+}^{n}\right)$ получаем

Следствие. Имеет место включение

$$
\sigma(T(t)) \supseteq e^{t \cdot \sigma_{T}(A)} \quad\left(t \in \mathbb{R}_{+}^{n}\right) .
$$

Теорема 2 вытекает из теорем 3 и 4 , устанавливаемых ниже.

\section{§3. Отображение коммутантного спектра}

Определение 7. Будем говорить, что точка $\lambda=\left(\lambda_{1}, \ldots, \lambda_{n}\right) \in \mathbb{C}^{n}$ является регулярной для набора $A$ (и писать $\lambda \in \rho_{\text {шा }}(A)$ ), если найдутся такие ограниченные операторы $B_{k}$ в $X, \operatorname{Im}\left(B_{k}\right) \subseteq D\left(A_{k}\right), k=1, \ldots, n$, коммутирующие со всеми $A_{j}$ (т. е. $B_{k} A_{j} \subseteq A_{j} B_{k}$ ), что

$$
\sum_{j=1}^{n}\left(\lambda_{j} I-A_{j}\right) B_{j}=I .
$$

Коммутантный спектр набора $A$ определим равенством ${ }^{1)} \sigma_{\amalg}(A)=\mathbb{C}^{n} \backslash$ $\rho_{\amalg}(A)$. зент).

1)Это понятие для случая ограниченных операторов введено в [17] (на это указал рецен- 
Очевидно, что в случае одного оператора $\sigma_{\text {II }}\left(A_{1}\right)$ совпадает с $\sigma\left(A_{1}\right)$.

Легко проверить также, что $\sigma_{\text {II }}(A) \subseteq \sigma\left(A_{1}\right) \times \cdots \times \sigma\left(A_{n}\right)$ (рассуждая, как в доказательстве предложения 1.9 из [17], можно показать, что $\sigma_{\text {II }}(A)$ есть замкнутое подмножество в $\mathbb{C}^{n}$, однако этот факт нам далее не понадобится).

Теорема 3. Для любой функиии Бернштейна $\psi$ от $n$ переменных справедливо включение

$$
\sigma(\psi(A)) \supseteq \psi(\sigma u(A)) .
$$

Доказательство. Докажем, что

$$
\rho_{\text {Шᄑ }}(A) \supseteq\left\{\lambda \in \mathbb{C}^{n}: \operatorname{Re} \lambda<0, \psi(\lambda) \in \rho(\psi(A))\right\} .
$$

При $\lambda \in \mathbb{C}^{n}, \operatorname{Re} \lambda<0$ и $x \in D(A)$ имеем в силу (1) и (3)

$$
\psi(\lambda) x-\psi(A) x=c_{1} \cdot(\lambda I-A) x+\int_{\mathbb{R}_{+}^{n}}\left(e^{\lambda \cdot u} I-T(u)\right) x d \mu(u) .
$$

Для любых ограниченных операторов $B_{j}, C_{j}, j=1, \ldots, n$, в $X$ справедливо тождество

$$
\prod_{j=1}^{n} B_{j}-\prod_{j=1}^{n} C_{j}=\left(B_{1}-C_{1}\right) \prod_{j=2}^{n} B_{j}+C_{1}\left(B_{2}-C_{2}\right) \prod_{j=3}^{n} B_{j}+\cdots+\prod_{j=1}^{n-1} C_{j}\left(B_{n}-C_{n}\right) .
$$

С учетом коммутирования полугрупп $T_{j}$ получаем отсюда, что

$$
e^{\lambda \cdot u} I-T(u)=\sum_{j=1}^{n}\left(e^{\lambda_{j} u_{j}} I-T_{j}\left(u_{j}\right)\right) U_{j}(u),
$$

где

$$
U_{j}(u)=\prod_{1 \leqslant l<j} T_{l}\left(u_{l}\right) \prod_{j<k \leqslant n} e^{\lambda_{k} u_{k}}
$$

- ограниченные операторы в $X$, коммутирующие с $T_{i}, i=1, \ldots, n,\left\|U_{j}(u)\right\| \leqslant$ $M^{n-1}$. Известно (см., например, [18, формулы (8.1a), (8.1b)]), что равенства

$$
V_{j}\left(u_{j}\right) x=\int_{0}^{u_{j}} e^{\left(u_{j}-s\right) \lambda_{j}} T_{j}(s) x d s
$$

определяют ограниченные операторы в $X, \operatorname{Im}\left(V_{j}\left(u_{j}\right)\right) \subseteq D\left(A_{j}\right)$, также коммутирующие со всеми $T_{i}$, причем $\left\|V_{j}\left(u_{j}\right) x\right\| \leqslant M\|x\|\left(e^{u_{j} \operatorname{Re} \lambda_{j}}-1\right) / \operatorname{Re} \lambda_{j}$ и

$$
\left(e^{\lambda_{j} u_{j}} I-T_{j}\left(u_{j}\right)\right) x=\left(\lambda_{j} I-A_{j}\right) V_{j}\left(u_{j}\right) x \quad \text { при } x \in X .
$$

Подставляя это в $(7)$, а результат - в $(6)$, имеем

$$
\begin{aligned}
(\psi(\lambda) I-\psi(A)) x & =\sum_{j=1}^{n} c_{1}^{j}\left(\lambda_{j} I-A_{j}\right) x+\sum_{j=1}^{n} \int_{\mathbb{R}_{+}^{n}}\left(\lambda_{j} I-A_{j}\right) V_{j}\left(u_{j}\right) U_{j}(u) x d \mu(u) \\
& =\sum_{j=1}^{n}\left(\lambda_{j} I-A_{j}\right) W_{j} x
\end{aligned}
$$

где операторы

$$
W_{j} x=c_{1}^{j} x+\int_{\mathbb{R}_{+}^{n}} V_{j}\left(u_{j}\right) U_{j}(u) x d \mu(u)
$$


ограничены в $X$, так как

$$
\begin{aligned}
\left\|W_{j} x\right\| & \leqslant\left(c_{1}^{j}+M^{n-1} \int_{\mathbb{R}_{+}^{n}}\left\|V_{j}\left(u_{j}\right)\right\| d \mu(u)\right)\|x\| \\
& \leqslant\left(c_{1}^{j}+\frac{M^{n}}{\operatorname{Re} \lambda_{j}} \int_{\mathbb{R}_{+}^{n}}\left(e^{u_{j} \operatorname{Re} \lambda_{j}}-1\right) d \mu(u)\right)\|x\|
\end{aligned}
$$

(интегралы сходятся, поскольку последний выражается через $\psi\left(\left(\operatorname{Re} \lambda_{j}\right) e_{j}\right)$, где $\left(e_{j}\right)_{j=1}^{n}-$ стандартный базис в $\left.\mathbb{R}^{n}\right)$. Кроме того, $\operatorname{Im}\left(W_{j}\right) \subseteq D\left(A_{j}\right)$ и $W_{j}$ коммутируют со всеми $T_{i}$, а потому и с $\psi(A)$.

Если $\psi(\lambda) \in \rho(\psi(A))$, то, заменяя в (8) $x$ на $(\psi(\lambda) I-\psi(A))^{-1} x, x \in X$, получаем

$$
x=\sum_{j=1}^{n}\left(\lambda_{j} I-A_{j}\right)\left(W_{j}(\psi(\lambda) I-\psi(A))^{-1}\right) x \quad \text { при } x \in X,
$$

т. е. $\lambda \in \rho_{\text {шI }}(A)$, что доказывает $(5)$.

Рассмотрим теперь случай, когда $\sigma\left(A_{j}\right) \subseteq\left\{\operatorname{Re} \lambda_{j}<0\right\}$ при всех $j$. Если мы предположим, что в этом случае теорема неверна, т. е. найдется число из $\psi\left(\sigma_{\text {ш }}(A)\right)$, не принадлежащее $\sigma(\psi(A))$, то $\psi(\lambda) \in \rho(\psi(A))$ при некотором $\lambda \in$ $\sigma_{\text {ш }}(A)$, а это противоречит $(5)$.

Перейдем к общему случаю. В силу ограниченности рассматриваемых полугрупп при всех $j=1, \ldots, n$ справедливо включение $\sigma\left(A_{j}\right) \subseteq\left\{\operatorname{Re} \lambda_{j} \leqslant 0\right\}$. Тогда для любого $\delta>0$ операторы $A_{j}-\delta I$ принадлежат $\operatorname{Gen}(X)$ и $\sigma\left(A_{j}-\delta I\right) \subseteq$ $\left\{\operatorname{Re} \lambda_{j}<0\right\}$ при всех $j$. Кроме того, $\psi_{\delta}(s):=\psi(s-\bar{\delta}) \in \mathscr{T}_{n}$ и $\psi_{\delta}(A)=\psi(A-\bar{\delta} I)$, где $\bar{\delta}:=(\delta, \ldots, \delta)$. По доказанному выше

$$
\sigma\left(\psi_{\delta}(A)\right)=\sigma(\psi(A-\bar{\delta} I)) \supseteq \psi\left(\sigma_{\amalg}(A-\bar{\delta} I)\right)=\psi_{\delta}(\sigma(\amalg(A)) .
$$

Далее, в доказательстве основной теоремы из [11] показано, что $\psi(A) x=$ $\psi_{\delta}(A) x+F_{\delta} x$ при $x \in D(A)$, где $F_{\delta}$ - ограниченный оператор. Но тогда $\psi(A)=$ $\psi_{\delta}(A)+F_{\delta}$, поскольку по теореме $1 D(A)$ есть существенная область для обеих частей этого равенства. Кроме того, в [11] показано, что $\left\|F_{\delta}\right\| \rightarrow 0(\delta \rightarrow 0)$, a потому по теореме о спектре возмущенного оператора (см. [19, теорема IV.3.6]; $\rho_{H}$ обозначает метрику Хаусдорфа)

$$
\rho_{H}\left(\sigma\left(\psi_{\delta}(A)\right), \sigma(\psi(A))\right) \rightarrow 0 \quad(\delta \rightarrow 0) .
$$

Следовательно, для любого $\varepsilon>0$ при достаточно малых $\delta>0$ имеем $\sigma\left(\psi_{\delta}(A)\right) \subseteq$ $B_{\varepsilon}[\sigma(\psi(A))]$; здесь $B_{\varepsilon}[N]:=\left\{z \in \mathbb{C}: \inf _{t \in N}|z-t| \leqslant \varepsilon\right\}$ для любого множества $N \subset \mathbb{C}$. А тогда $\psi_{\delta}\left(\sigma_{\amalg}(A)\right) \subseteq B_{\varepsilon}[\sigma(\psi(A))]$ в силу $(9)$.

$\mathrm{C}$ другой стороны, при достаточно малых $\delta>0$ имеет место включение $\psi\left(\sigma_{\text {ш }}(A)\right) \subseteq B_{\varepsilon}\left[\psi_{\delta}\left(\sigma_{\amalg}(A)\right)\right]$. Это следует из того, что $\psi(\lambda)-\psi(\lambda-\bar{\delta}) \rightarrow 0(\delta \rightarrow 0)$ равномерно по $\lambda \in\{\operatorname{Re} \lambda \leqslant 0\}$, так как

$$
|\psi(\lambda)-\psi(\lambda-\bar{\delta})| \leqslant c_{1} \cdot \bar{\delta}+\int_{\mathbb{R}_{+}^{n}}\left(1-e^{-u \cdot \bar{\delta}}\right) d \mu(u) \rightarrow 0 \quad(\delta \rightarrow+0)
$$

по теореме Б. Леви. Следовательно, $\psi\left(\sigma_{\amalg}(A)\right) \subseteq B_{\varepsilon}\left[B_{\varepsilon}[\sigma(\psi(A))]\right] \subseteq B_{2 \varepsilon}[\sigma(\psi(A))]$, 
а потому

$$
\psi\left(\sigma_{\amalg}(A)\right) \subseteq \bigcap_{\varepsilon>0} B_{2 \varepsilon}[\sigma(\psi(A))]=\sigma(\psi(A))
$$

в силу замкнутости множества $\sigma(\psi(A))$.

\section{§4. Сравнение коммутантного спектра и спектра Тейлора - окончание доказательства теоремы 2}

Основная теорема вытекает из теоремы 3 и следующего утверждения.

Теорема 4. Имеет место включение

$$
\sigma_{\text {ШII }}(A) \supseteq \sigma_{T}(A) .
$$

Для доказательства нам понадобятся две леммы.

Лемма 2. Пусть ограниченный оператор $B: X \rightarrow X$ коммутирует с каждым из операторов $T_{k}(t)$. Тогда $B$ отображсает линейное многообразие $D\left(A_{i_{1}} \cdots A_{i_{m}}\right)$ в себя для любого набора индексов $1 \leqslant i_{1}<\cdots<i_{m} \leqslant n u$

$$
B A_{i_{1}} \cdots A_{i_{m}} x=A_{i_{1}} B A_{i_{2}} \cdots A_{i_{m}} x=\cdots=A_{i_{1}} \cdots A_{i_{m}} B x
$$

для любого $x \in D\left(A_{i_{1}} \cdots A_{i_{m}}\right)$.

Доказательство. Оператор $B$ коммутирует с каждым из генераторов $A_{k}$, откуда следует справедливость утверждения леммы при $m=1$. Предположим теперь, что $m \geqslant 2$, и покажем, что $B A_{i_{2}} \cdots A_{i_{m}} x \in D\left(A_{i_{1}}\right)$ при любом $x \in$ $D\left(A_{i_{1}} \cdots A_{i_{m}}\right)$ и

$$
B A_{i_{1}} \cdots A_{i_{m}} x=A_{i_{1}} B A_{i_{2}} \cdots A_{i_{m}} x
$$

Для доказательства достаточно заметить, что для вектора $y:=A_{i_{2}} \cdots A_{i_{m}} x$, принадлежащего $D\left(A_{i_{1}}\right)$,

$$
\frac{T_{i_{1}}(h) B y-B y}{h}=B \frac{T_{i_{1}}(h) y-y}{h},
$$

причем правая часть стремится к $B A_{i_{1}} y$ при $h \rightarrow 0$.

Применяя теперь доказанное утверждение к $B A_{i_{2}} \cdots A_{i_{m}} x$ и т. д. (и учитывая коммутирование $B$ с $\left.A_{k}\right)$, получаем требуемое.

Лемма 3. Пусть ограниченный оператор $B: X \rightarrow X$ коммутирует с каждъмм из операторов $A_{j}, j=1, \ldots, n, u \operatorname{Im}(B) \subseteq D\left(A_{k}\right)$ для некоторого $k$. Тогда для любого набора индексов $1 \leqslant j_{1}<\cdots<j_{m} \leqslant n$ справедливо включение

$$
\begin{aligned}
& D\left(A_{j_{1}} \cdots A_{j_{m}}\right) \\
& \quad \subseteq D\left(A_{k} A_{j_{1}} \cdots A_{j_{m}} B\right) \cap D\left(A_{j_{1}} A_{k} A_{j_{2}} \cdots A_{j_{m}} B\right) \cap \cdots \cap D\left(A_{j_{1}} \cdots A_{j_{m}} A_{k} B\right) .
\end{aligned}
$$

При этом, если $x \in D\left(A_{j_{1}} \cdots A_{j_{m}}\right)$, то

$$
A_{k} A_{j_{1}} \cdots A_{j_{m}} B x=A_{j_{1}} A_{k} A_{j_{2}} \cdots A_{j_{m}} B x=\cdots=A_{j_{1}} \cdots A_{j_{m}} A_{k} B x .
$$

Доказательство леммы проведем индукцией по $m$.

Рассмотрим сначала случай $m=1$. Пусть $x \in D\left(A_{j_{1}}\right)$. Тогда $A_{j_{1}} B x=$ $B A_{j_{1}} x \in D\left(A_{k}\right)$, т. е. $x \in D\left(A_{k} A_{j_{1}} B\right)$. Kроме того, $B x \in D\left(A_{k} A_{j_{1}}\right) \cap D\left(A_{k}\right) \subseteq$ $D\left(A_{j_{1}} A_{k}\right)$ по лемме 1. Значит, $x \in D\left(A_{j_{1}} A_{k} B\right)$ и по той же лемме $A_{j_{1}} A_{k} B x=$ $A_{k} A_{j_{1}} B x$.

Переходя к случаю $m=2$, предположим, что $x \in D\left(A_{j_{1}} A_{j_{2}}\right)$. Тогда по лемме 2 $A_{k} B A_{j_{1}} A_{j_{2}} x=A_{k} A_{j_{1}} B A_{j_{2}} x=A_{k} A_{j_{1}} A_{j_{2}} B x$, причем все значения существуют. 
Следовательно, $x \in D\left(A_{k} A_{j_{1}} A_{j_{2}} B\right)$ и, кроме того, $B A_{j_{2}} x \in D\left(A_{k} A_{j_{1}}\right) \cap D\left(A_{k}\right)$. Поэтому по лемме $1 A_{j_{2}} B x=B A_{j_{2}} x \in D\left(A_{j_{1}} A_{k}\right)$, т. е. $x \in D\left(A_{j_{1}} A_{k} A_{j_{2}} B\right)$. Снова применяя лемму 1 , выводим отсюда, что $B x \in D\left(A_{k} A_{j_{2}}\right) \cap D\left(A_{k}\right) \subseteq D\left(A_{j_{2}} A_{k}\right)$

и $A_{k} A_{j_{2}} B x=A_{j_{2}} A_{k} B x$. А так как существует $A_{j_{1}} A_{k} A_{j_{2}} B x$, то существует и $A_{j_{1}} A_{j_{2}} A_{k} B x$ и справедливы равенства

$$
A_{k} A_{j_{1}} A_{j_{2}} B x=A_{j_{1}} A_{k} A_{j_{2}} B x=A_{j_{1}} A_{j_{2}} A_{k} B x,
$$

что доказывает наше утверждение при $m=2$.

Предположим теперь, что утверждение леммы верно при некотором $m \geqslant 2$, a $x \in D\left(A_{j_{1}} \cdots A_{j_{m+1}}\right)$. Тогда $x_{1}:=A_{j_{3}} \cdots A_{j_{m+1}} x \in D\left(A_{j_{1}} A_{j_{2}}\right)$ и в силу рассмотренного выше случая $m=2$

$$
A_{k} A_{j_{1}} A_{j_{2}} B x_{1}=A_{j_{1}} A_{k} A_{j_{2}} B x_{1}=A_{j_{1}} A_{j_{2}} A_{k} B x_{1},
$$

причем все значения существуют. Но по лемме $2 B x_{1}=A_{j_{3}} \cdots A_{j_{m+1}} B x$. С учетом (10) отсюда следует, что

$$
A_{k} A_{j_{1}} A_{j_{2}} \cdots A_{j_{m+1}} B x=A_{j_{1}} A_{k} A_{j_{2}} \cdots A_{j_{m+1}} B x=A_{j_{1}} A_{j_{2}} A_{k} A_{j_{3}} \cdots A_{j_{m+1}} B x
$$

и все значения существуют. А так как $x \in D\left(A_{j_{3}} \cdots A_{j_{m+1}}\right)$, то по индуктивному предположению

$$
A_{k} A_{j_{3}} \cdots A_{j_{m+1}} B x=A_{j_{3}} A_{k} \cdots A_{j_{m+1}} B x=\cdots=A_{j_{3}} \cdots A_{j_{m+1}} A_{k} B x,
$$

где все значения также существуют. Подстановка этих равенств в (11) завершает индуктивный переход.

Доказательство теоремы 4. Достаточно показать, что обобщенный комплекс Кошуля $\mathscr{K}(X, \lambda-A)$ точен для любого $\lambda \in \rho_{\text {ш }}(A)$. Пусть $B_{k}-$ операторы в $X$, фигурирующие в определении 7 . Как и для случая ограниченных операторов (см. [20, гл. 1, предложение 2]), положим

$$
s_{m}: X_{m} \rightarrow X_{m+1}: x \otimes e_{i_{1}} \wedge \cdots \wedge e_{i_{m}} \mapsto-\sum_{k=1}^{n} B_{k} x \otimes e_{k} \wedge e_{i_{1}} \wedge \cdots \wedge e_{i_{m}}, \quad x \in X .
$$

Точность комплекса $\mathscr{K}(X, \lambda-A)$ будет следовать из справедливости равенств

$$
d_{m}(\lambda-A) s_{m} y+s_{m-1} d_{m-1}(\lambda-A) y=y \quad(m=1, \ldots, n)
$$

при всех $y \in D\left(d_{m-1}(A)\right)$. Формально они проверяются непосредственно, однако, в отличие от случая ограниченных операторов, мы должны убедиться еще в том, что $s_{m} y \in D\left(d_{m}(A)\right)$ (напомним, что $\left.D\left(d_{m}(\lambda-A)\right)=D\left(d_{m}(A)\right)\right)$. Но если $y=x \otimes e_{i_{1}} \wedge \cdots \wedge e_{i_{m}}$, где $x \in D\left(A_{j_{1}} \cdots A_{j_{m}}\right)$ для любой перестановки $\left(j_{1}, \ldots, j_{m}\right)$ мультииндекса $\left(i_{1}, \ldots, i_{m}\right)$, то по лемме 3 для любого $k=1, \ldots, n$

$$
B_{k} x \in D\left(A_{k} A_{j_{1}} \cdots A_{j_{m}}\right) \cap D\left(A_{j_{1}} A_{k} A_{j_{2}} \cdots A_{j_{m}}\right) \cap \cdots \cap D\left(A_{j_{1}} \cdots A_{j_{m}} A_{k}\right),
$$

т. е. $B_{k} x \in D\left(A_{l_{1}} \cdots A_{l_{m+1}}\right)$ для любой перестановки $\left(l_{1}, \ldots, l_{m+1}\right)$ мультииндекса $\left(k, i_{1}, \ldots, i_{m}\right)$. Последнее и означает, что $s_{m} y \in D\left(d_{m}(A)\right)$.

Отметим, что включение в теореме 4 может быть строгим даже в случае ограниченных операторов (см. [21, теорема 4.1]).

Автор благодарит рецензента за замечания, способствовавшие улучшению изложения. 


\section{ЛитеРАтУРА}

[1] A. Kishimoto, D. Robinson, Subordinate semigroups and order properties, J. Austral. Math. Soc. Ser. A, 31:1 (1981), 59-76.

[2] Ch. Berg, K. Boyadzhiev, R. deLaubenfels, Generation of generators of holomorphic semigroups, J. Austral. Math. Soc. Ser. A, 55:2 (1993), 246-269.

[3] A. S. Carasso, T. Kato, On subordinated holomorphic semigroups, Trans. Amer. Math. Soc., 327:2 (1991), 867-878.

[4] А. Р. Миротин, $O \mathscr{T}$-исчислении генераторов $C_{0}$-полугрупп, Сиб. матем. ж., 39:3 (1998), 571-582.

[5] R. Shilling, R. Song, Z. Vondraček, Bernstein Functions. Theory and Applications, de Greyter, Berlin-New York, 2010.

[6] В. Феллер, Введение в теорию вероятностей и ее приложения, т. 2, Мир, М., 1984.

[7] Э. Хилле, Р. Филлипс, Функциональный анализ и полугруппы, ИЛ, М., 1962.

[8] D. Applebaum, Levy processes - from probability to finance and quantum groups, Notices Amer. Math. Soc., 51:11 (2004), 1336-1347.

[9] А. Р. Миротин, Действие функиий класса Шенберга Я та конусе диссипативных элементов банаховой алгебры, Матем. заметки, 61:4 (1997), 630-633.

[10] А. Р. Миротин, Функиии класса Шенберга $\mathscr{T}$ действуют в конусе диссипативных элементов банаховой алгебры, II, Матем. заметки, 64:3 (1998), 423-430.

[11] А. Р. Миротин, Многомерное $\mathscr{T}$-исчисление от генераторов $C_{0}$-полугрупп, Алгебра и анализ, 11:2 (1999), 142-170.

[12] Ch. Berg, J. P. R. Christensen, P. Ressel, Harmonic Analysis on Semigroups, Graduate Texts in Math., vol. 100, Springer-Verlag, New York-Berlin, 1984.

[13] А. Р. Миротин, О многомерном функциональном исчислении Бохнера-Филлипса, Проблемы физики, математики и техники, 1:1 (2009), 63-66.

[14] У. Браттели, Д. Робинсон, Операторные алгебры и квантовая статистическая механика, Мир, М., 1982.

[15] Дж. Голдстейн, Полугруппы линейных операторов и их приложения, Высшая школа, Киев, 1989.

[16] А. Я. Хелемский, Гомология в банаховых и топологических алгебрах, Изд-во МГУ, М., 1986.

[17] Z. Slodkowski, W. Zelazko, On joint spectra of commuting families of operators, Studia Math., 50 (1974), 127-148.

[18] Ф. Клемент, Х. Хейманс, С. Ангенент и др., Однопараметрические полугруппы, Мир, М., 1992.

[19] Т. Като, Теория возмущений линейных операторов, Мир, М., 1972.

[20] А. Я. Хелемский, Гомологические методы в голоморфном исчислении от нескольких операторов в банаховом пространстве, по Тейлору, УМН, 36:1(217) (1981), $127-172$.

[21] J. L. Taylor, A joint spectrum for several commuting operators, J. Funct. Anal., 6:2 (1970), 172-191.

Гомельский государственный университет имени Ф. Скорины

Поступило в редакцию e-mail: amirotin@yandex.ru 